PROCEEDINGS OF THE

AMERICAN MATHEMATICAL SOCIETY

Volume 140, Number 5, May 2012, Pages 1535-1547

S 0002-9939(2011)11020-7

Article electronically published on September 21, 2011

\title{
CHARACTERIZATION OF EXTREMAL VALUED FIELDS
}

\author{
SALIH AZGIN, FRANZ-VIKTOR KUHLMANN, AND FLORIAN POP
}

(Communicated by Bernd Ulrich)

\begin{abstract}
We characterize those valued fields for which the image of the valuation ring under every polynomial in several variables contains an element of maximal value or zero.
\end{abstract}

\section{INTRODUCTION}

The notion of extremality for valued fields was introduced by Yuri Ershov in [4] in connection with valued skew fields which are finite-dimensional over their center. It turns out that the original definition given in that paper (and also in talks given by its author) is flawed in the sense that there are no extremal valued fields except algebraically closed valued fields, and Proposition 2 of that paper is false. We fix this flaw by slightly modifying the definition of extremality; see Definition 3.1 below.

The notion of extremality, restricted to certain classes of polynomials, has since become very useful for the characterization of various properties of valued fields; cf. 9 .

In valuation theory and particularly the model theory of valued fields, power series fields and, more generally, maximal fields (valued fields without proper immediate extensions) are usually known to have very good properties. For instance, all of them are henselian, and what is more, algebraically complete. So it seemed likely that all of them are also extremal. Our results in this paper will show that this is not the case.

In the present paper, we obtain the following characterization of extremal valued fields with residue characteristic 0 :

Theorem 1.1. Extremal valued fields with residue characteristic 0 are precisely

(i) henselian valued fields whose value group is a $\mathbb{Z}$-group, and

(ii) henselian valued fields whose value group is divisible and whose residue field is large.

Received by the editors July 1, 2009 and, in revised form, September 22, 2009; April 11, 2010; and January 20, 2011.

2010 Mathematics Subject Classification. Primary 12J10; Secondary 12E30.

The second author was partially supported by a Canadian NSERC grant and by a sabbatical grant of the University of Saskatchewan.

The third author was supported by the John Templeton Foundation grant ID 13394.

A major part of this research was done while the authors were attending the o-minimality program at the Fields Institute, January-June 2009. The authors would like to thank the Fields Institute for its support and hospitality.

(C)2011 American Mathematical Society Reverts to public domain 28 years from publication 
More generally, we prove in Section 4

Theorem 1.2. Let $\mathcal{K}=(K, \Gamma, \boldsymbol{k} ; v)$ be a valued field. If $\mathcal{K}$ is extremal, then $\mathcal{K}$ is algebraically complete and

(i) $\Gamma$ is a $\mathbb{Z}$-group, or

(ii) $\Gamma$ is divisible and $\boldsymbol{k}$ is large.

If $K$ is perfect with char $\boldsymbol{k}=$ char $K$, then the converse also holds.

It remains an open question whether the above characterization also holds in the case of mixed characteristic. In the case of nonperfect valued fields, it does not hold. While for every field $k$, the formal Laurent Series Field $k((t))$ with its canonical valuation $v_{t}$ is extremal according to Theorem 4.1 below, we will show in Section 4:

Theorem 1.3. There exist nonextremal algebraically complete valued fields of equal positive characteristic with value group a $\mathbb{Z}$-group and such that under a coarsening of their valuation, they are still not extremal, have divisible value group and nonperfect large residue field.

See the following section for the definitions of algebraically complete valued field, $\mathbb{Z}$-group and large field.

We also prove that every finite extension of an extremal field is again extremal (Theorem [3.10). In a recent paper [5], Ershov has modified his definition of "extremal" in the same way we do and has proved this result with a different approach.

\section{Preliminaries}

We assume familiarity with the basic concepts of valued fields and their modeltheoretic properties. We consider valued fields as three-sorted structures

$$
\mathcal{K}=(K, \Gamma, \boldsymbol{k} ; v),
$$

where $K$ is the underlying field, $\Gamma$ is the value group, $k$ is the residue field, $v$ : $K^{\times} \rightarrow \Gamma$ is the valuation, with valuation ring

$$
\mathcal{O}_{v}:=\{a \in K: v(a) \geq 0\}
$$

and maximal ideal $\mathfrak{m}_{v}:=\{a \in K: v(a)>0\}$ of $\mathcal{O}$. We have the residue class map

$$
\begin{gathered}
{ }^{-}: \mathcal{O}_{v} \rightarrow \boldsymbol{k}, \\
a \mapsto a+\mathfrak{m}_{v},
\end{gathered}
$$

and the residue characteristic of $K$ is the characteristic of $\boldsymbol{k}$. We often refer to $K$ as the valued field, instead of $\mathcal{K}$. When there is more than one valuation defined on the same field, we use the notation $K v$ and $v K$ to denote the residue field and value group of $K$ respectively for the valuation $v$. We also use the notation $a v$ to denote the residue class of an element in $\mathcal{O}_{v}$. For a subset $\Delta$ of an ordered group $\Gamma$ we use the notation

$$
\gamma<\Delta
$$

as a shorthand for $\gamma<\delta$ for all $\delta \in \Delta$. We set $v(0):=\infty>\Gamma, \Gamma_{\infty}:=\Gamma \cup\{\infty\}$, $-\infty<\Gamma$, and for $\gamma \in \Gamma$ the intervals $(-\infty, \gamma)$ and $(\gamma, \infty)$ are defined as usual. We use $m, n, \ldots$ to denote elements of $\mathbb{N}$ unless specified otherwise. 
Definition 2.1. A valued field $\mathcal{K}$ is called algebraically maximal if it does not admit proper immediate algebraic extensions (that is, extensions which preserve the value group and the residue field). Since henselizations are immediate algebraic extensions, every algebraically maximal field is henselian.

Definition 2.2. A valued field $\mathcal{K}$ is called algebraically complete if it is henselian and for every finite extension $(L, \Delta, \boldsymbol{l} ; w)$ we have

$$
[L: K]=(\Delta: \Gamma)[\boldsymbol{l}: \boldsymbol{k}] \text {. }
$$

If equation (2.1) holds, then the extension is called defectless.

Definition 2.3. An ordered group is regular if for each $n$ every open interval that contains $n$ elements contains an $n$-divisible element. A $\mathbb{Z}$-group is a regular group which is discrete, i.e., has a smallest positive element. An ordered group is dense if for any two elements $\alpha<\beta$ there is an element $\gamma$ in the group such that $\alpha<\gamma<\beta$.

Note that $\mathbb{Z}$-groups are exactly the ordered groups which are elementarily equivalent to $\mathbb{Z}$. If $\Gamma$ is dense and regular, then for each $n$ and $\gamma \in \Gamma$ there is an increasing sequence $\left\{n \gamma_{\rho}\right\}_{\rho<\lambda}$ which is cofinal in $(-\infty, \gamma)$. We state two useful facts on regular groups from [13] and 11 respectively.

Theorem 2.4. A regular group is either a $\mathbb{Z}$-group or it is dense.

Theorem 2.5. An ordered group $\Gamma$ is regular if and only if $\Gamma / \Delta$ is divisible for every nonzero convex subgroup $\Delta$ of $\Gamma$.

Definition 2.6. Take $\mathcal{A} \subseteq \mathcal{B}$ to be an extension of two structures of a first order language. Then $\mathcal{A}$ is existentially closed in $\mathcal{B}$ if every existential sentence with parameters from $\mathcal{A}$ that holds in $\mathcal{B}$ also holds in $\mathcal{A}$.

Definition 2.7. A field $\boldsymbol{k}$ is large if every smooth curve over $k$ which has a $k$ rational point has infinitely many such points.

Algebraically closed fields, real closed fields, pseudo-algebraically closed fields, and fields equipped with a henselian valuation are all large fields. Finite fields are not large. Note that a field $\boldsymbol{k}$ is large if and only if it is existentially closed in $\boldsymbol{k}((t))$ ([11; see also [8]).

For the following result, see Theorem 17 of [8].

Theorem 2.8. Suppose that $\boldsymbol{k}$ is a large and perfect subfield of a field $F$. If $\boldsymbol{k}$ is the residue field of a valuation on $F$ which is trivial on $\boldsymbol{k}$, then $\boldsymbol{k}$ is existentially closed in F (as a field).

Finally, we will need the following two well-known technical lemmas.

Lemma 2.9. Let $\mathcal{K}=(K, \Gamma, \boldsymbol{k} ; v)$ be a valued field such that $v=w \circ \bar{w}$. Then $\mathcal{K}$ is henselian if and only if $w$ is henselian on $K$ and $\bar{w}$ is henselian on the residue field $K w$ of $K$ under $w$. The same holds for "algebraically complete" in the place of "henselian".

By the use of Hensel's Lemma, one proves:

Lemma 2.10. Let $\mathcal{K}=(K, \Gamma, \boldsymbol{k} ; v)$ be a perfect and henselian valued field. Then every embedding of a subfield of $\boldsymbol{k}$ such that $v$ is trivial on the image can be extended to an embedding of $\boldsymbol{k}$ such that $v$ is trivial on the image. 


\section{Properties of extremal Fields}

Definition 3.1. A valued field $\mathcal{K}$ is extremal if for every multi-variable polynomial $F\left(X_{1}, \ldots, X_{n}\right)$ over $K$ the set

$$
\left\{v\left(F\left(a_{1}, \ldots, a_{n}\right)\right): a_{1}, \ldots, a_{n} \in \mathcal{O}_{v}\right\} \subseteq \Gamma_{\infty}
$$

has a maximal element.

Remark 3.2. The original definition presented in [4] asks for a maximal element of the set

$$
\left\{v\left(F\left(a_{1}, \ldots, a_{n}\right)\right): a_{1}, \ldots, a_{n} \in K\right\} \subseteq \Gamma_{\infty},
$$

where $K$ and $F$ are as above. This condition is not satisfied for the polynomial

$$
F(X, Y)=X^{2}+(X Y-1)^{2}
$$

over the Laurent series field $\mathbb{R}((t))$ as

$$
v\left(F\left(t^{n}, t^{-n}\right)\right)=2 n
$$

and $F(a, b) \neq 0$ for all $a, b \in \mathbb{R}((t))$. Hence, Proposition 2 of [4] does not hold for the original definition.

Suppose that $K$ is a valued field which is not algebraically closed. Take a polynomial $f(x)=x^{n}+a_{n-1} x^{n-1}+\cdots+a_{0} \in K[x]$ with no zeros in $K$ and let

$$
G(X, Y)=X^{n}+a_{n-1} X^{n-1} Y+\cdots+a_{0} Y^{n}
$$

be its homogenization. Then the polynomial $F(X, Y)=G(X, X Y-1)$ does not satisfy the above condition. Indeed, $G(X, Y)$ can only be zero if $Y$ is zero and then, consequently, also $X$ is zero. So $F(X, Y)$ can only be zero if $X Y-1$ and $X$ are zero, which is impossible. On the other hand, for $a \neq 0$ we have that $v F\left(a, a^{-1}\right)=v\left(a^{n}\right)=n v(a)$, which shows that $\{v(F(a, b) \mid a, b \in K\}$ has no maximum.

The following is a consequence of Theorem 1.5 of [9]:

Proposition 3.2. Every extremal field is algebraically maximal and hence henselian.

Proposition 3.3. Let $\mathcal{K}=(K, \Gamma, \boldsymbol{k} ; v)$ be a valued field with $\Gamma$ dense and regular. If $\mathcal{K}$ is extremal, then $\Gamma$ is divisible.

Proof. Let $\gamma \in \Gamma$ be positive and not divisible by $n>1$. Consider the polynomial

$$
F(x, y)=x^{4 n}+\epsilon\left(x y-\epsilon^{2}\right)^{n}+\epsilon^{2} y^{4 n}
$$

over $K$ where $v(\epsilon)=\gamma$. Note that for all $a, b \in K^{\times}$,

$$
v\left(a^{4 n}\right), \quad v\left(\epsilon\left(a b-\epsilon^{2}\right)^{n}\right), \quad v\left(\epsilon^{2} b^{4 n}\right)
$$

are distinct elements of $\Gamma_{\infty}$ since $\gamma$ is not $n$-divisible. Hence the valuation of $F(a, b)$ is equal to the minimum of the three distinct elements above.

We claim that for all $a, b \in \mathcal{O}_{v}, v(F(a, b))<4 n \gamma+\gamma$. Assume otherwise. Then $v\left(a^{4 n}\right) \geq 4 n \gamma+\gamma$ and since equality is not possible,

$$
4 n v(a)>4 n \gamma+\gamma \text {. }
$$

Also, $v\left(\epsilon\left(a b-\epsilon^{2}\right)^{n}\right) \geq 4 n \gamma+\gamma$ and so $v(a)+v(b)=2 \gamma$. Consequently,

$$
4 n v(b)=8 n \gamma-4 n v(a) \text {, }
$$


which gives $4 n v(b)<4 n \gamma-\gamma$. Then

$$
v(F(a, b)) \leq v\left(\epsilon^{2} b^{4 n}\right)=2 \gamma+4 n v(b)<4 n \gamma+\gamma,
$$

a contradiction.

Since $\Gamma$ is dense and regular we can take a sequence $\left\{4 n \delta_{\rho}\right\}_{\rho<\lambda}$ in $\Gamma$ which is cofinal in the interval $(4 n \gamma, 4 n \gamma+\gamma)$. For each $\rho<\lambda$, pick $a_{\rho} \in \mathcal{O}_{v}$ with $v\left(a_{\rho}\right)=\delta_{\rho}<2 \gamma$ and let $b_{\rho}:=\epsilon^{2} a_{\rho}^{-1} \in \mathcal{O}_{v}$. Then for all $\rho$,

$$
v\left(a_{\rho}^{4 n}\right)=4 n \delta_{\rho}<4 n \gamma+\gamma<v\left(\epsilon^{2} b_{\rho}^{4 n}\right)=2 \gamma+4 n\left(2 \gamma-\delta_{\rho}\right)
$$

and hence $v\left(F\left(a_{\rho}, b_{\rho}\right)\right)=4 n \delta_{\rho}$. Together with the previous claim, this shows that $\left\{v(F(a, b)): a, b \in \mathcal{O}_{v}\right\}$ has no maximal element and so $\mathcal{K}$ is not extremal.

Proposition 3.4. Let $\mathcal{K}=(K, \Gamma, \boldsymbol{k}, v)$ be an extremal valued field. Then for each nonzero convex subgroup $\Delta$ of $\Gamma$, the quotient group $\Gamma / \Delta$ is divisible.

Proof. Let $\gamma \in \Gamma$ be such that the coset $\gamma+\Delta$ is not divisible by $n$ in $\Gamma / \Delta$. Consider the polynomial (as introduced in the previous theorem)

$$
F(x, y)=x^{4 n}+\epsilon\left(x y-\epsilon^{2}\right)^{n}+\epsilon^{2} y^{4 n}
$$

over $K$, where $v(\epsilon)=\gamma$.

The arguments in the proof of Proposition 3.3 can be applied to the ordered group $\Gamma / \Delta$ to conclude that for all $a, b \in \mathcal{O}_{v}$,

$$
v(F(a, b))<4 n \gamma+\gamma+\Delta .
$$

Claim. $\left\{v\left(F(a, b): a, b \in \mathcal{O}_{v}\right\}\right.$ has no maximal element. Take $a, b \in \mathcal{O}_{v}$ and set $\theta=v(F(a, b))$. We aim to find $a^{\prime}, b^{\prime} \in \mathcal{O}_{v}$ with $v\left(F\left(a^{\prime}, b^{\prime}\right)\right)>\theta$. Take any positive $\delta \in \Delta$. From (3.1) it follows that

$$
\theta+4 n \delta<4 n \gamma+\gamma-4 n \delta .
$$

Note that $v(F(\epsilon, \epsilon))=4 n \gamma$, so we assume that $4 n \gamma<\theta$. As $v(\epsilon)=\gamma$ is not divisible by $n, \theta$ is equal to the minimum of $4 n v(a), \gamma+n v\left(a b-\epsilon^{2}\right)$ and $2 \gamma+4 n v(b)$. Therefore,

$$
4 n \gamma<\theta \leq v\left(\epsilon\left(a b-\epsilon^{2}\right)^{n}\right)
$$

and hence

$$
v(b)=2 \gamma-v(a) .
$$

Assume that $\theta=4 n v(a)$. Together with $4 n v(a)<4 n \gamma+\gamma$, (3.2) shows that

$$
4 n v(a)+4 n \delta<\gamma+4 n \gamma-4 n \delta<2 \gamma+8 n \gamma-4 n v(a)-4 n \delta .
$$

Now take $a^{\prime} \in \mathcal{O}_{v}$ with $v\left(a^{\prime}\right)=v(a)+\delta$ and let $b^{\prime}=\epsilon^{2} / a^{\prime} \in \mathcal{O}_{v}$. Then $v\left(b^{\prime}\right)=$ $2 \gamma-v(a)-\delta$ and (3.3) implies that $4 n v\left(a^{\prime}\right)<2 \gamma+4 n v\left(b^{\prime}\right)$. Therefore $v\left(F\left(a^{\prime}, b^{\prime}\right)\right)=$ $4 n v\left(a^{\prime}\right)=4 n v(a)+4 n \delta>\theta$, as required.

Next assume that $\theta=\gamma+n v\left(a b-\epsilon^{2}\right) \geq 4 n \gamma$. Then $\theta<4 n v(a)$ and $\theta<$ $2 \gamma+4 n v(b)$. With $b^{\prime}=\epsilon^{2} / a \in \mathcal{O}_{v}$, we have $v\left(b^{\prime}\right)=v(b)$ and therefore $v\left(F\left(a, b^{\prime}\right)\right) \geq$ $\min \left\{4 n v(a), 2 \gamma+4 n v\left(b^{\prime}\right)\right\}>\theta$.

It remains to consider the case $\theta=2 \gamma+4 n v(b)$. Together with $2 \gamma+4 n v(b)=$ $\theta<4 n \gamma+\gamma,(3.2)$ shows that

$$
\begin{aligned}
2 \gamma+4 n v(b)+4 n \delta & <4 n \gamma+\gamma-4 n \delta+(4 n \gamma+\gamma-2 \gamma-4 n v(b))-4 n \delta \\
& =8 n \gamma-4 n v(b)-4 n \delta .
\end{aligned}
$$


Now take $b^{\prime} \in \mathcal{O}_{v}$ with $v\left(b^{\prime}\right)=v(b)+\delta$ and let $a^{\prime}=\epsilon^{2} / b^{\prime} \in \mathcal{O}_{v}$. Then $v\left(a^{\prime}\right)=$ $2 \gamma-v(b)-\delta$, and the above inequality implies that $2 \gamma+4 n v\left(b^{\prime}\right)<4 n v\left(a^{\prime}\right)$. Therefore $v\left(F\left(a^{\prime}, b^{\prime}\right)\right)=2 \gamma+4 n v(b)+4 n \delta>\theta$, as required. This completes the proof of our claim, and we conclude that $\mathcal{K}$ is not extremal.

Theorem 3.5. Let $\mathcal{K}=(K, \Gamma, \boldsymbol{k} ; v)$ be an extremal valued field. Then $\Gamma$ is divisible or a $\mathbb{Z}$-group.

Proof. Proposition 3.4 shows that $\Gamma$ has no nonzero convex subgroup $\Delta$ such that $\Gamma / \Delta$ is not divisible. Therefore, $\Gamma$ is regular by Theorem 2.5. If $\Gamma$ is not a $\mathbb{Z}$-group, then, by Theorem 2.4 $\Gamma$ is dense and Proposition 3.3 yields that $\Gamma$ is divisible.

Proposition 3.6. Let $\mathcal{K}=(K, \Gamma, \boldsymbol{k} ; v)$ be an extremal valued field with nonalgebraically closed residue field $\boldsymbol{k}$ and divisible value group $\Gamma$. Then there is no polynomial $f(Y, Z)$ over $\mathcal{O}_{v}$ such that

(i) there are $\alpha_{1}, \ldots, \alpha_{m} \in \boldsymbol{k}$ such that the equation

$$
\bar{f}(\alpha, z)=0
$$

over $\boldsymbol{k}$ has no solution in $\boldsymbol{k}$ whenever $\alpha \neq \alpha_{1}, \ldots, \alpha_{m}$;

(ii) for all $\epsilon \in \mathfrak{m}_{v}, f(\epsilon, Z)=0$ has a solution in $\mathcal{O}_{v}$.

Proof. Let $\beta_{0}, \ldots, \beta_{n-1} \in \boldsymbol{k}$ be such that $x^{n}+\cdots+\beta_{1} x+\beta_{0}=0$ has no solutions in $\boldsymbol{k}$. Consider the polynomial

$$
F(X, Y)=X^{n}+\cdots+b_{1} X Y^{n-1}+b_{0} Y^{n}
$$

over $\mathcal{O}_{v}$, where $\bar{b}_{i}=\beta_{i}$ and $b_{i}=0$ if $\beta_{i}=0$, for $i=0, \ldots, n-1$. Note that for all positive $\gamma \in \Gamma$,

$$
v(F(a, b)) \geq \gamma \Longrightarrow v(a), v(b) \geq \gamma / n
$$

Let $G(X, Y, Z):=F(F(X, Y), Z)$. For positive $\gamma \in \Gamma$, if $v(G(a, b, c)) \geq \gamma$, then $v(a), v(b) \geq \gamma / n^{2}$ and $v(c) \geq \gamma / n$.

Let $a_{1}, \ldots, a_{m} \in \mathcal{O}_{v}$ be such that $\bar{a}_{j}=\alpha_{j}$ and $a_{j}=0$ if $\alpha_{j}=0$ (where $\alpha_{j}$ is as in (i) above) for $j=1, \ldots, m$. Consider the polynomial

$$
H(X, Y, Z):=G\left(X, f(Y, Z), X Y\left(Y-a_{1}\right) \cdots\left(Y-a_{m}\right)-\epsilon\right)
$$

over $K$ where $v(\epsilon)=\gamma>0$. We claim that

$$
v(H(a, b, c))<n^{2} \gamma
$$

for all $a, b, c \in \mathcal{O}_{v}$. Otherwise, we would have

$$
v(a) \geq \gamma \text { and } v\left(a b\left(b-a_{1}\right) \cdots\left(b-a_{m}\right)-\epsilon\right) \geq n \gamma .
$$

Therefore $v\left(a b\left(b-a_{1}\right) \cdots\left(b-a_{m}\right)\right)=v(\epsilon)=\gamma$, which in turn gives

$$
v(a)=\gamma, \quad v(b)=0 \text { and } v\left(b-a_{i}\right)=0 \quad \text { for } \quad i=1, \ldots, m .
$$

So $\bar{b} \neq \alpha_{1}, \ldots, \alpha_{m}$, and hence for all $z \in \mathcal{O}_{v}, \bar{f}(\bar{b}, \bar{z}) \neq 0$. Then $v(H(a, b, c))=$ $v(f(b, c))=0$, a contradiction, and we establish the claim.

Let $\left\{\gamma_{\rho}\right\}_{\rho<\lambda}$ be a decreasing sequence such that $\gamma_{\rho} \rightarrow 0$ and pick $b_{\rho} \in \mathfrak{m}_{v}$ with $v\left(b_{\rho}\right)=\gamma_{\rho}$ for all $\rho<\lambda$. Let $a_{\rho}$ be such that $a_{\rho} b_{\rho}\left(b_{\rho}-a_{1}\right) \ldots\left(b_{\rho}-a_{m}\right)=\epsilon$. Note that $v\left(a_{\rho}\right) \rightarrow \gamma$, and in particular we may assume that $a_{\rho} \in \mathcal{O}_{v}$ for all $\rho<\lambda$. Pick $c_{\rho} \in \mathcal{O}_{v}$ such that $f\left(b_{\rho}, c_{\rho}\right)=0$ for each $\rho<\lambda$. Then

$$
v\left(H\left(a_{\rho}, b_{\rho}, c_{\rho}\right)\right)=n^{2} v\left(a_{\rho}\right)
$$


and hence $\left\{v\left(H\left(a_{\rho}, b_{\rho}, c_{\rho}\right)\right)\right\}$ is cofinal in $\left(0, n^{2} \gamma\right)$. We conclude that $\mathcal{K}$ is not extremal.

Theorem 3.7. Suppose that $\mathcal{K}=(K, \Gamma, \boldsymbol{k} ; v)$ has a divisible value group. If $\mathcal{K}$ is extremal, then $\boldsymbol{k}$ is large.

Proof. Suppose that $\boldsymbol{k}$ is not large. Then there is a curve $C$ defined over $\boldsymbol{k}$ which has a smooth $\boldsymbol{k}$-rational point but has only finitely many $\boldsymbol{k}$-rational points. But then by the theory of algebraic curves, the curve $C$ is birationally equivalent to a curve in the affine $\boldsymbol{k}$-plane $C_{h}=V(h) \subset \mathbf{A}_{\boldsymbol{k}}^{2}$, where $h=h(Y, Z) \in \boldsymbol{k}[Y, Z]$ is a polynomial in the variables $Y, Z$ satisfying the following:

(i) ${ }^{\prime} C_{h}(\boldsymbol{k})=\left\{(\alpha, \beta) \in \boldsymbol{k}^{2} \mid h(\alpha, \beta)=0\right\}$ is finite.

(ii) $^{\prime} h(0,0)=0$ and $\partial h / \partial Z(0,0) \neq 0$; hence in particular, $(0,0)$ is a smooth $k$-rational point of $C_{h}$. (Actually these conditions imply that $h(Y, Z)$ has the form $h(Y, Z)=\gamma_{10} Y+\gamma_{01} Z+$ (nonlinear terms) with $\gamma_{01} \neq 0$.)

Setting $h(Y, Z)=\sum_{i, j} \gamma_{i j} Y^{i} Z^{j}$, let $f(Y, Z)=\sum_{i, j} c_{i j} Y^{i} Z^{j} \in \mathcal{O}[Y, Z]$ be a preimage of $h(Y, Z)$ such that $c_{i j}=0$ if $\gamma_{i j}=0$; hence $c_{i j} \in \mathcal{O}^{\times}$if $\gamma_{i j} \neq 0$. Finally, let $\left\{\alpha_{1}=0, \ldots, \alpha_{m}\right\}$ be the set of all the $Y$-coordinates of the points $(\alpha, \beta) \in C_{h}(\boldsymbol{k})$. We claim that $f(Y, Z) \in \mathcal{O}[Y, Z]$ satisfies the conditions (i), (ii) from Proposition 3.6. Indeed, condition (i) is obviously satisfied, because $\bar{f}(Y, Z)=$ $h(Y, Z)$, and $h(Y, Z)$ satisfies condition (i)'. For condition (ii), proceed as follows: For $\epsilon \in \mathfrak{m}_{v}$ set $f_{\epsilon}(Z):=f(\epsilon, Z) \in \mathcal{O}[Z]$. Then $\bar{f}_{\epsilon}(Z)=h(0, Z)$; hence $Z=0$ is a simple zero of $\bar{f}_{\epsilon}(Z)=h(0, Z)$ by condition (ii)'. Therefore, by Hensel's Lemma there exists a unique $\eta \in \mathfrak{m}_{v}$ such that $f_{\epsilon}(\eta)=0$; hence equivalently, $f(\epsilon, \eta)=f_{\epsilon}(\eta)=0$.

By Proposition 3.6 we conclude that $\mathcal{K}$ is not extremal.

The results proved so far enable us to give a version of Proposition 1 of [4] for the modified notion of "extremal field".

Definition 3.8. We say that a basis $b_{1}, \ldots, b_{n}$ of a valued field extension

$$
(K, \Gamma, \boldsymbol{k} ; v) \subset(L, \Delta, \boldsymbol{l} ; w)
$$

is a valuation basis if for all choices of $c_{1}, \ldots, c_{n} \in K$,

$$
w \sum_{i=1}^{n} c_{i} b_{i}=\min _{i} w c_{i} b_{i} .
$$

Note that every finite defectless extension admits a valuation basis.

Lemma 3.9. Every finite defectless extension of an extremal field is again an extremal field.

Proof. Take an extremal field $\mathcal{K}=(K, \Gamma, \boldsymbol{k} ; v)$ and a finite defectless extension $(L, \Delta, \boldsymbol{l} ; w)$ of degree $m$; we wish to show that the latter is an extremal field. From Theorem 3.5 we know that $\Gamma$ is divisible or a $\mathbb{Z}$-group. Therefore, all cosets of $\Gamma$ in $\Delta$ admit representatives that are either 0 or lie between 0 and the smallest positive element of $\Gamma$. Consequently, we can choose a valuation basis $b_{1}, \ldots, b_{m}$ of the extension such that the values $w b_{i}$ have the same property. Write $\bar{Y}=\left(Y_{1}, \ldots, Y_{m}\right)$ and take

$$
h(\bar{Y})=N_{L(\bar{Y}) \mid K(\bar{Y})}\left(\sum_{i=1}^{m} b_{i} Y_{i}\right)
$$


to be the norm form with respect to the basis $b_{1}, \ldots, b_{m}$ of the extension $L(\bar{Y}) \mid$ $K(\bar{Y})$.

Take a polynomial $F\left(X_{1}, \ldots, X_{n}\right)$ over $L$; we wish to show that the set

$$
\left\{w\left(F\left(a_{1}, \ldots, a_{n}\right)\right): a_{1}, \ldots, a_{n} \in \mathcal{O}_{w}\right\}
$$

has a maximal element. Denote by $G(\bar{Z})$ the polynomial obtained from $F$ by substituting $\sum_{i=1}^{m} b_{i} Z_{i j}$ for $X_{i}, 1 \leq i \leq n$. The polynomial $G(\bar{Z})$ can be written as $G(\bar{Z})=\sum_{i=1}^{m} b_{i} G_{i}(\bar{Z})$ with $G_{i}(\bar{Z}) \in K[\bar{Z}]$ for every $i$. Now let

$$
H(\bar{Z})=h\left(G_{1}(\bar{Z}), \ldots, G_{m}(\bar{Z})\right)=N_{L(\bar{Y}) \mid K(\bar{Y})}(G(\bar{Z})) \in K[\bar{Z}] .
$$

Since $\mathcal{K}$ is extremal, there exist elements $c_{i j} \in \mathcal{O}_{v}, 1 \leq i \leq m, 1 \leq j \leq n$, such that

$$
v H(\bar{c})=\max \left\{w H\left(\overline{c^{\prime}}\right) \mid c_{i j}^{\prime} \in \mathcal{O}_{v}\right\} .
$$

For $1 \leq j \leq n$, we set $d_{j}=\sum_{i=1}^{m} b_{i} c_{i j}$. We wish to show that

$$
w F(\bar{d})=\max \left\{w F\left(\overline{d^{\prime}}\right) \mid d_{j}^{\prime} \in \mathcal{O}_{w}\right\} .
$$

Note that $v N_{L \mid K}(a)=m w a$ for every $a \in L$ since $\mathcal{K}$ is henselian by Proposition 3.2. Take $d_{j}^{\prime} \in \mathcal{O}_{w}$ and write $d_{j}^{\prime}=\sum_{i=1}^{m} b_{i} c_{i j}^{\prime}$ with $c_{i j}^{\prime} \in K$. Since the $b_{i}$ form a valuation basis,

$$
0 \leq v d_{j}^{\prime}=w \sum_{i=1}^{m} b_{i} c_{i j}^{\prime}=\min _{i} w b_{i} c_{i j}^{\prime} .
$$

Hence for $1 \leq i \leq m$ and $1 \leq j \leq n, w b_{i}+v c_{i j}^{\prime} \geq 0$. By our assumptions on the values $w b_{i}$, this implies that $c_{i j}^{\prime} \in \mathcal{O}_{v}$. Now we compute

$$
\begin{aligned}
w F\left(\overline{d^{\prime}}\right) & =\frac{1}{m} v N_{L \mid K}\left(F\left(\overline{d^{\prime}}\right)\right)=\frac{1}{m} v H\left(\overline{c^{\prime}}\right) \\
& \leq \frac{1}{m} v H(\bar{c})=\frac{1}{m} v N_{L \mid K}(F(\bar{d}))=w F(\bar{d}) .
\end{aligned}
$$

This lemma yields:

Theorem 3.10. a) Every extremal valued field is algebraically complete.

b) Every finite extension of an extremal field is again an extremal field.

Proof. a): By Proposition 3.2, every extremal field is algebraically maximal. Hence our assertion follows from the preceding lemma once we know that a valued field is algebraically complete if each of its finite defectless extensions is algebraically maximal. This holds by Corollary 2.10 of 9 . For the convenience of the reader, we give a sketch of the proof. Every finite extension of an algebraically complete field is again algebraically complete, hence algebraically maximal. For the converse, take a valued field $\mathcal{K}$ such that every finite extension is algebraically maximal. Then $\mathcal{K}$ is itself algebraically maximal, hence henselian. Take a finite extension $\mathcal{L}$ of $\mathcal{K}$. In order to show that it is defectless, it suffices to show that its normal hull $\mathcal{N}$ is a defectless extension of $\mathcal{K}$. Denote by $\mathcal{N}^{\prime}$ the maximal separable subextension of $\mathcal{N} \mid \mathcal{K}$ and by $\mathcal{R}$ the ramification field of this Galois extension. Then $\mathcal{R} \mid \mathcal{K}$ is defectless. On the other hand, $\mathcal{N}^{\prime} \mid \mathcal{K}$ is a $p$-extension, where $p$ is the characteristic of the residue field. Consequently, $\mathcal{N} \mid \mathcal{K}$ is a tower of (separable or purely inseparable) extensions of degree $p$. As $\mathcal{R} \mid \mathcal{K}$ is defectless, there is a maximal field $\mathcal{L}^{\prime}$ in the tower such that $\mathcal{L}^{\prime} \mid \mathcal{K}$ is defectless. By assumption, $\mathcal{L}^{\prime}$ is algebraically maximal. If $\mathcal{L}^{\prime} \neq \mathcal{N}$, then 
the next larger field $\mathcal{L}^{\prime \prime}$ in the tower is an extension of degree $p$ of $\mathcal{L}^{\prime}$ and it is not immediate, hence defectless. By multiplicativity of the defect, we find that $\mathcal{L}^{\prime \prime} \mid \mathcal{K}$ is defectless, contradicting the maximality of $\mathcal{L}^{\prime}$.

b): By part a), every finite extension of an extremal field is defectless. Hence the assertion of part b) follows from the preceding lemma.

\section{Characterization of extremal fields}

The first nonalgebraically closed examples of extremal fields were provided by Proposition 2 in [4]:

Theorem 4.1. Let $\mathcal{K}=(K, \Gamma, \boldsymbol{k} ; v)$ be an algebraically complete valued field with $\Gamma \simeq \mathbb{Z}$. Then $\mathcal{K}$ is extremal.

By Remark 3.2, this theorem would be false with the definition of extremality as stated in [4. However, it is easy to check that the proof of Theorem 4.1]given in [4] is valid with the revised definition of extremality. In fact, the corrected argument to be inserted in that proof is exactly the argument used at the beginning of Case 1 of the proof of Theorem 4.4 below.

Note that extremality is a first order condition for valued fields. Hence we can apply the following Ax-Kochen-Ershov Principle for tame valued fields proved in [6] (see also [10]) to show extremality for larger classes of valued fields:

Theorem 4.2. Let $\mathcal{K}=(K, \Gamma, \boldsymbol{k} ; v)$ and $\mathcal{L}=(L, \Delta, \boldsymbol{l} ; w)$ be two perfect algebraically complete valued fields with $\operatorname{char} \boldsymbol{k}=\operatorname{char} K$ and $\operatorname{char} \boldsymbol{l}=\operatorname{char} L$. If $\boldsymbol{k} \equiv \boldsymbol{l}$ and $\Gamma \equiv \Delta$, then $\mathcal{K} \equiv \mathcal{L}$. If in addition $\mathcal{L}$ is an extension of $\mathcal{K}$ and if $\boldsymbol{k} \prec \boldsymbol{l}$ and $\Gamma \prec \Delta$, then $\mathcal{K} \prec \mathcal{L}$. The same holds with " ${ }_{\exists}$ " in the place of " $\prec$ " as soon as $\mathcal{K}$ is perfect and algebraically complete with $\operatorname{char} \boldsymbol{k}=\operatorname{char} K$.

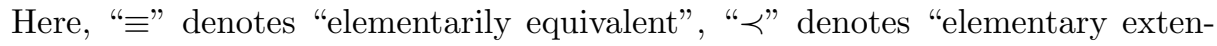
sion" and " $\prec \exists "$ denotes "existentially closed in".

If the residue field has characteristic 0 , then "perfect algebraically complete" is equivalent to "henselian"; this is a consequence of the Lemma of Ostrowski. As a corollary to Theorems 4.1 and 4.2 we obtain:

Theorem 4.3. Let $\mathcal{K}=(K, \Gamma, \boldsymbol{k} ; v)$ be a henselian valued field with $\Gamma$ a $\mathbb{Z}$-group and $\operatorname{char} \boldsymbol{k}=\operatorname{char} K=0$. Then $\mathcal{K}$ is extremal.

The following theorem includes also the case of fields of positive characteristic:

Theorem 4.4. Let $\mathcal{K}=(K, \Gamma, \boldsymbol{k} ; v)$ be a perfect algebraically complete valued field with divisible value group $\Gamma$ and large residue field $\boldsymbol{k}$ with $\operatorname{char} \boldsymbol{k}=\operatorname{char} K$. Then $\mathcal{K}$ is extremal.

Proof. In view of Theorem 4.2, we only have to prove our theorem in the case where $\Gamma$ is the ordered additive group of the real numbers. Take any polynomial $F \in K\left[X_{1}, \ldots, X_{n}\right]$. Take $\mathcal{K}^{*}=\left(K^{*}, \Gamma^{*}, \boldsymbol{k}^{*} ; v^{*}\right)$ to be a $|K|^{+}$-saturated elementary extension of $\mathcal{K}$. Then $\Gamma^{*}$ is an elementary extension of $\Gamma$, and $\boldsymbol{k}^{*}$ is an elementary extension of $\boldsymbol{k}$. Now we distinguish two cases. 
Case 1. $\left\{v\left(F\left(a_{1}, \ldots, a_{n}\right)\right): a_{1}, \ldots, a_{n} \in \mathcal{O}_{v}\right\} \backslash\{\infty\}$ is cofinal in $\Gamma$. We wish to show that $F$ has a zero in $\mathcal{O}_{v}^{n}$

By our choice of $\mathcal{K}^{*}$ there are $a_{1}^{*}, \ldots, a_{n}^{*} \in \mathcal{O}_{v^{*}}$ such that $v^{*}\left(F\left(a_{1}^{*}, \ldots, a_{n}^{*}\right)\right)>$ $\Gamma$. Hence there exists a valuation $w$ on $\mathcal{K}^{*}$ that is coarser than $v^{*}$, satisfies $w F\left(a_{1}^{*}, \ldots, a_{n}^{*}\right)>0$, but is trivial on $K$. So we can consider $K$ as a subfield of the residue field $K^{*} w$ of $K^{*}$ under $w$. We write $v^{*}=w \circ \bar{w}$, where $\bar{w}$ is the valuation induced by $v^{*}$ on $K^{*} w$. Note that $a_{1}^{*}, \ldots, a_{n}^{*} \in \mathcal{O}_{w}$. We obtain that $0=F\left(a_{1}^{*}, \ldots, a_{n}^{*}\right) w=F\left(a_{1}^{*} w, \ldots, a_{n}^{*} w\right)$ and that $a_{1}^{*} w, \ldots, a_{n}^{*} w \in \mathcal{O}_{\bar{w}}$. Denote the value group of $\bar{w}$ on $K^{*} w$ by $\Gamma^{\prime}$. Since $\Gamma$ is divisible, $\Gamma \prec_{\exists} \Gamma^{\prime}$. Further, $\boldsymbol{k}^{*}$ is equal to the residue field of $K^{*} w$ under $\bar{w}$, and $\boldsymbol{k} \prec_{\exists} \boldsymbol{k}^{*}$. Hence by Theorem 4.2, $\mathcal{K} \prec_{\exists}\left(K^{*} w, \Gamma^{\prime}, \boldsymbol{k}^{*} ; \bar{w}\right)$. Since $F$ has a zero in the valuation ring of the latter field, this implies that there are $a_{1}, \ldots, a_{n} \in \mathcal{O}_{v}$ such that $F\left(a_{1}, \ldots, a_{n}\right)=0$.

Case 2. $\left\{v\left(F\left(a_{1}, \ldots, a_{n}\right)\right): a_{1}, \ldots, a_{n} \in \mathcal{O}_{v}\right\}$ is not cofinal in $\Gamma=\mathbb{R}$. Then there is some real number $r$ which is the supremum of this set. We wish to show that it is a member of the set.

By our choice of $\mathcal{K}^{*}$ there are $a_{1}^{*}, \ldots, a_{n}^{*} \in \mathcal{O}_{v^{*}}$ such that $\delta^{*}:=v^{*}\left(F\left(a_{1}^{*}, \ldots, a_{n}^{*}\right)\right)$ $\geq v\left(F\left(a_{1}, \ldots, a_{n}\right)\right)$ for all $a_{1}, \ldots, a_{n} \in \mathcal{O}_{v}$. On the other hand, $\delta^{*} \leq r$ in $\Gamma^{*}$. So $0 \leq r-\delta^{*}<s$ for all positive $s \in \mathbb{R}=\Gamma$. We take $\Gamma_{0}$ to be the largest convex subgroup of $\Gamma^{*}$ such that $\Gamma_{0} \cap \Gamma=\{0\}$. Then we take $w$ to be the coarsening of $v^{*}$ with respect to $\Gamma_{0}$; that is, $\mathcal{O}_{w}$ is generated over $\mathcal{O}_{v}$ by all elements whose values under $v$ lie in $\Gamma_{0}$, and the value group of $w$ on $K^{*}$ is $\Gamma^{*} / \Gamma_{0}$. Again, we write $v^{*}=w \circ \bar{w}$, where $\bar{w}$ is the valuation induced by $v^{*}$ on the residue field $K^{*} w$. We observe that $v=w$ on $K$ (after identification of equivalent valuations). Further, $w\left(F\left(a_{1}^{*}, \ldots, a_{n}^{*}\right)\right)=r$.

Now we have that $\left(K^{*}, \Gamma^{*} / \Gamma_{0}, K^{*} w ; w\right)$ is an extension of $\mathcal{K}$. We have canonical embeddings of $\boldsymbol{k}$ in $K^{*} w$ and of $\Gamma$ in $\Gamma^{*} / \Gamma_{0}$. Since $\Gamma$ is divisible, $\Gamma \prec_{\exists} \Gamma^{*} / \Gamma_{0}$. The residue field $K^{*} w$ has a valuation $\bar{w}$ with residue field $\boldsymbol{k}^{*}$. Being an elementary extension of $K, K^{*}$ is also perfect. It follows that its residue fields $K^{*} w$ and $\boldsymbol{k}^{*}$ are perfect. Since every algebraically complete valued field is henselian, Lemma 2.9 shows that $K^{*} w$ is henselian under $\bar{w}$. By Lemma 2.10 the canonical embedding of $\boldsymbol{k}$ in $K^{*} w$ can be extended to an embedding of $\boldsymbol{k}^{*}$ in $K^{*} w$. Via this embedding we may assume that $\boldsymbol{k}^{*}$ is a subfield of $K^{*} w$. Since $\boldsymbol{k}^{*}$ is an elementary extension of $\boldsymbol{k}$, it is again a large field. Thus by Theorem 2.8 $\boldsymbol{k}^{*} \prec_{\exists} K^{*} w$, and consequently, $\boldsymbol{k} \prec_{\exists} K^{*} w$. It now follows from Theorem 4.2 that $\mathcal{K} \prec_{\exists}\left(K^{*}, \Gamma^{*} / \Gamma_{0}, K^{*} w ; w\right)$. Hence there are $a_{1}, \ldots, a_{n} \in \mathcal{O}_{v}$ such that $v F\left(a_{1}, \ldots, a_{n}\right)=r$.

Theorem 1.2 now follows from Theorems $4.3,4.4,3.5,3.7$ and 3.10 ,

We will now give an example that will prove Theorem 1.3. Its construction is given in the paper 7]; we will use the same notation as in that paper. Taking the valued field $(K, v)$ appearing in the construction to be $\left(\mathbb{F}_{p}((t)), v_{t}\right)$, where $\mathbb{F}_{p}$ is the field with $p$ elements and $v_{t}$ is the canonical $t$-adic valuation on $\mathbb{F}_{p}((t))$, we obtain a valued field extension $(L, v)$ of $\left(\mathbb{F}_{p}((t)), v_{t}\right)$ with the following properties:

a) $L \mid \mathbb{F}_{p}((t))$ is a regular extension of transcendence degree 1 ,

b) $(L, v)$ is algebraically complete,

c) the value group $v L$ is a $\mathbb{Z}$-group with smallest positive element $v(t)$,

d) the residue field $L v$ is equal to $\mathbb{F}_{p}$,

e) $v=w \circ v_{t}$ for the finest valuation $w$ that is coarser than $v$,

f) the value group $w L=\mathbb{Q}$ is divisible and by Lemma 2.9, $(L, w)$ is algebraically complete, 
g) the residue field $L w=\mathbb{F}_{p}((t))$ is a large field as it carries a henselian valuation,

h) for the polynomial $G\left(X_{0}, X_{1}\right)=X_{0}^{p}-X_{0}+t X_{1}^{p}-x$ (where $x=s^{-1}$ in the notation of [7]), $w\left(G\left(x_{0}, x_{1}\right)\right)<0$ for all $x_{0}, x_{1} \in L$ (because it is shown in [7] that if $w\left(G\left(x_{0}, x_{1}\right)\right) \geq 0$, then $x_{1}$ must be transcendental over $\left.L\right)$.

The equality $L w=\mathbb{F}_{p}((t))$ holds because in the construction, $(L, w)$ is an immediate extension of a field $\left(L_{2}, w\right)$ with residue field $L_{2} w=K=\mathbb{F}_{p}((t))$.

Using the notation of [7], in particular the recursive definition

$$
\xi_{1}=x^{1 / p} \quad \text { and } \quad \xi_{j+1}=\left(\xi_{j}-c_{1} s^{-p / q_{j}}\right)^{1 / p}
$$

with $c_{1}=t$, and setting

$$
a_{k}=\sum_{j=1}^{k} \xi_{j} \quad \text { and } \quad b_{k}=\sum_{j=1}^{k-1} s^{-1 / q_{j}}
$$

we compute:

$$
\begin{aligned}
x-\left(a_{k}^{p}-a_{k}\right)-c_{1} b_{k}^{p} & =x-\sum_{j=1}^{k}\left(\xi_{j}^{p}-\xi_{j}\right)-c_{1} \sum_{j=1}^{k-1} s^{-p / q_{j}} \\
& =x-\xi_{1}^{p}-\sum_{j=1}^{k-1}\left(\xi_{j+1}^{p}-\xi_{j}\right)+\xi_{k}-c_{1} \sum_{j=1}^{k-1} s^{-p / q_{j}} \\
& =\sum_{j=1}^{k-1} c_{1} s^{-p / q_{j}}+\xi_{k}-\sum_{j=1}^{k-1} c_{1} s^{-p / q_{j}} \\
& =\xi_{k} .
\end{aligned}
$$

Hence,

$$
w\left(G\left(a_{k}, b_{k}\right)\right)=w \xi_{k}=-\frac{1}{p^{k}} w s<0 .
$$

This shows that $\left\{w\left(G\left(a_{k}, b_{k}\right)\right) \mid k \in \mathbb{N}\right\}$ is cofinal in the negative part $w L^{<0}$ of $w L$.

By the definition of $a_{k}$, we have $w\left(a_{k}\right)=w\left(\xi_{1}\right)=\frac{1}{p} w(x)=-\frac{1}{p} w(s)<0$ for all $k$. Similarly, $w\left(b_{k}\right)=w\left(s^{-1 / q_{1}}\right)=-\frac{1}{q_{1}} w(s)<0$ for all $k$. Consequently, $w\left(s a_{k}\right)>0$, $w\left(s b_{k}\right)>0$ and hence $s a_{k}, s b_{k} \in \mathcal{O}_{v} \subset \mathcal{O}_{w}$. Now set

$$
F(X, Y)=G\left(s^{-1} X, s^{-1} Y\right)=s^{-p} X^{p}-s^{-1} X+t s^{-p} Y^{p}-s^{-1} .
$$

Then $w\left(F\left(s a_{k}, s b_{k}\right)\right)=w\left(G\left(a_{k}, b_{k}\right)\right)$, but we still have that $w(F(a, b))<0$ for all $a, b \in L$. So we see that $\left\{w(F(a, b)) \mid a, b \in \mathcal{O}_{w}\right\}$ is a cofinal subset of $w L^{<0}$ and thus has no maximal element. This proves that $(L, w)$ is not extremal.

Observe that $w(F(a, b))<0$ is equivalent to $v(F(a, b))<v_{t} \mathbb{F}_{p}((t))$. As the set $\left\{w(F(a, b)) \mid a, b \in \mathcal{O}_{w}\right\}$ is a cofinal subset of $w L^{<0}$, the set $\left\{v(F(a, b)) \mid a, b \in \mathcal{O}_{v}\right\}$ is a cofinal subset of the set of all values $<v_{t} \mathbb{F}_{p}((t))$ in $v L$ and thus has no maximal element. This proves that $(L, v)$ is not extremal.

\section{SOME FURTHER RESUlts}

An Ax-Kochen-Ershov Principle as in Theorem 4.2 also holds for formally $\wp-$ adic fields (see [12]) and, more generally, for finitely ramified fields (see [3], 14]). A formally $\wp$-adic field is $\wp$-adically closed if and only if it is henselian and its value group is a $\mathbb{Z}$-group. Formally $\wp$-adic and finitely ramified fields are algebraically 
complete as soon as they are henselian. Hence, we obtain from Theorems 4.1 and 1.1 via the Ax-Kochen-Ershov Principle:

Theorem 5.1. A formally $\wp$-adic field is extremal if and only if it is $\wp$-adically closed. A finitely ramified field is extremal if and only if it is henselian and its value group is a $\mathbb{Z}$-group.

If $\mathcal{K}=(K, \Gamma, \boldsymbol{k} ; v)$ is a valued field such that $v=w \circ \bar{w}$, then by Lemma 2.9, $v$ is henselian if and only if $w$ and $\bar{w}$ are. The same holds for "algebraically complete" in the place of "henselian". The corresponding assertion for "extremal" is not entirely known. We leave the easy proof of the following result to the reader:

Lemma 5.2. If $\mathcal{K}$ is extremal, then $K$ is also extremal with respect to every coarsening $w$ of $v$.

From our characterization of extremal fields we obtain:

Proposition 5.3. Let $\mathcal{K}=(K, \Gamma, \boldsymbol{k} ; v)$ be a perfect algebraically complete valued field such that $\operatorname{char} \boldsymbol{k}=\operatorname{char} K$ and $v=w \circ \bar{w}$. Then $\mathcal{K}=(K, \Gamma, \boldsymbol{k} ; v)$ is extremal if and only if $K$ is extremal with respect to $w$ and the residue field of $K$ under $w$ is extremal with respect to $\bar{w}$.

\section{ACKNOWLEDGEMENT}

The authors would like to thank Sergei Starchenko for pointing out the flaw in the definition of extremality and for providing the first example given in Remark 3.2.

\section{REFERENCES}

1. Paul F. Conrad. Regularly ordered groups. Proc. Amer. Math. Soc. 13 (1962), 726-731. MR0146272 (26:3794)

2. Françoise Delon. Quelques propriétés des corps valués en théories des modèles. Thèse de doctorat d'état, Paris 7 (1982).

3. Yu. L. Ershov. On the elementary theory of maximal valued fields, III. Algebra Logika 6 (1967), 31-38. MR0223231(36:6280)

4. Yu. L. Ershov. Extremal valued fields. Algebra Logika 43 (2004), 582-588, 631. Translation in Algebra Logic 43 (2004), 327-330. MR2112060 (2005m:12009)

5. Yu. L. Ershov. ^-extremal valued fields. Sibirsk. Mat. Zh. 50 (2009), 1280-1284. MR2603869

6. F.-V. Kuhlmann. Henselian Function Fields. Ph.D. Thesis, Heidelberg (1989), available at http://math.usask.ca/ffvk/diss.pdf

7. F.-V. Kuhlmann. Elementary properties of power series fields over finite fields. J. Symb. Logic 66 (2001), 771-791. MR1833477 (2002e:03057)

8. F.-V. Kuhlmann. Places of algebraic function fields in arbitrary characteristic. Advances in Math. 188 (2004), 399-424. MR2087232 (2005h:12014)

9. F.-V. Kuhlmann. A classification of Artin-Schreier defect extensions and a characterization of defectless fields. To appear in Illinois J. Math.

10. F.-V. Kuhlmann. The model theory of tame valued fields. In preparation. Preliminary version published in: Structures Algébriques Ordonnées, Séminaire 2007-2008, Paris VII (2009).

11. Florian Pop. Embedding problems over large fields, Annals of Math. (2) 144 (1996), 1-34. MR:1405941 (97h:12013)

12. Alexander Prestel and Peter Roquette. Formally $p$-adic fields. Lecture Notes in Math. 1050, Berlin-Heidelberg-New York-Tokyo (1984). MR738076 (85m:11090) 
13. Abraham Robinson and Elias Zakon. Elementary properties of ordered abelian groups. Trans. Amer. Math. Soc. 96 (1960), 222-236. MR0114855 (22:5673)

14. Martin Ziegler. Die elementare Theorie der henselschen Körper. Inaugural Dissertation, Köln (1972).

Department of Mathematics and Statistics, McMaster University, 1280 Main Street West, Hamilton, L8S 4K1 Ontario, Canada

E-mail address: sazgin@math.mcmaster.ca

Current address: Middle Eastern Technical University, Northern Cyprus Campus, Kalkanl, Güzelyurt, KKTC, Mersin 10, Turkey

E-mail address: sazgin@metu.edu.tr

Department of Mathematics and Statistics, University of Saskatchewan, 106 Wiggins Road, Saskatoon, Saskatchewan, Canada S7N 5E6

E-mail address: fvk@math.usask.ca

Department of Mathematics, University of Pennsylvania, 209 South 33rd Street, Philadelphia, Pennsylvania 19104-6395

E-mail address: pop@math.upenn.edu 